\title{
The COLOFOL trial: study design and comparison of the study population with the source cancer population
}

This article was published in the following Dove Press journal:

Clinical Epidemiology

28 January 2016

Number of times this article has been viewed

Pernilla Hansdotter

Andersson'

Peer Wille-Jørgensen ${ }^{2}$

Erzsébet Horváth-Puhó ${ }^{3}$

Sune Høirup Petersen ${ }^{2}$

Anna Martling ${ }^{4}$

Henrik Toft Sørensen ${ }^{3}$

Ingvar Syk'

\section{On behalf of the COLOFOL Study Group}

'Department of Surgery, Skåne University Hospital, Malmö, Sweden; ${ }^{2}$ Abdominal Disease Center K, Bispebjerg University Hospital, Copenhagen, Denmark; ${ }^{3}$ Department of Clinical Epidemiology, Aarhus University Hospital, Aarhus, Denmark; ${ }^{4}$ Department of Molecular Medicine and Surgery, Karolinska Institutet, Solna, Sweden
Correspondence: Ingvar Syk

Department of Surgery, Skåne University Hospital, 20502 Malmö, Sweden

Tel +46 40333742

Fax +46 40927877

Email ingvar.syk@telia.com
Introduction: The COLOFOL trial, a prospective randomized multicenter trial comparing two follow-up regimes after curative surgical treatment for colorectal cancer, focuses on detection of asymptomatic recurrences. This paper aims to describe the design and recruitment procedure in the COLOFOL trial, comparing demographic characteristics between randomized patients and eligible patients not included in the study.

Materials and methods: COLOFOL was designed as a pragmatic trial with wide inclusion criteria and few exclusion criteria, in order to obtain a sample reflecting the general patient population. To be eligible, patients had to be 75 years or younger and curatively resected for stage II or III colorectal cancer. Exclusion criteria were hereditary colorectal cancer, no signed consent, other malignancy, and life expectancy less than 2 years due to concomitant disease. In four of the 24 participating centers, we scrutinized hospital inpatient data to identify all colorectal cancer patients who underwent surgery, in order to ascertain all eligible patients who were not included in the study and to compare them with enrolled patients.

Results: Of a total of 4,445 eligible patients, 2,509 patients were randomized $(56.4 \%$ inclusion rate). A total of 1,221 eligible patients were identified in the scrutinized hospitals, of which 684 (56\%) were randomized. No difference in age or sex distribution was observed between randomized and nonrandomized eligible patients. However, a difference was noted in tumor location and stage distribution, with 5.6\% more patients in the randomized group having colon cancer and $6.7 \%$ more patients having stage II disease.

Conclusion: Patients in the two study arms were not only demographically similar, but also similar to nonincluded eligible patients, apart from stage and localization. The analyses will be stratified by these variables. Taken together, we conclude that our trial results will be robust and possible to extrapolate to the target population.

Keywords: trial design, source population, colorectal cancer, follow-up

\section{Introduction}

Follow-up after colorectal cancer surgery has three purposes: patient support, monitoring, and detection of asymptomatic recurrences to allow treatment with curative intent. However, it is not clear whether scheduled examinations and visits to the outpatient clinic have any survival benefit. ${ }^{1}$ Existing trials have been too small to be able to detect a difference between arms. The majority were also conducted in the era before modern imaging techniques and/or before availability of modern multimodal treatment of metastatic disease..$^{2-8}$

Meta-analyses of six randomized trials have indicated that more intense follow-up programs have a survival benefit. ${ }^{9,10}$ However, the heterogeneity of the trials calls their results into question. For example, the control group in one trial involved more 
examinations than the exposed group in another trial. ${ }^{5,6}$ Two of these trials showed a positive effect on survival. However, in one of them, this was explained by allocation of more patients with stage III disease to the control group. ${ }^{4}$ In the other trial, all re-resections were performed on local recurrences, which were very frequent (19\% and $25 \%$, respectively), ${ }^{8}$ indicating suboptimal primary treatment.

Thus, there is a strong need for a large randomized trial to evaluate different follow-up regimens for patients treated for colorectal cancer. The COLOFOL trial, launched in 2005, is a pragmatic trial focusing on detection of potentially curable recurrences. It evaluates two follow-up schedules in patients who underwent surgery for colorectal cancer with curative intent (http://www.colofol.com). Primary outcomes are overall survival and disease-free survival. By December 2010, target enrollment was reached, and recruitment was stopped. The trial is registered in the Clinical Trials Register (NCT00225641).

A randomized controlled trial represents the most unbiased method to compare different treatments between groups, because it prevents unmeasured and unknown confounding. However, trial inclusion procedures create the risk that the study population does not reflect the target population, due to too narrow inclusion criteria and/or nonrandom exclusion of eligible nonparticipants. ${ }^{11}$ This leads to subsequent difficulty in extrapolating the results to the general patient population and might explain why trial-based treatment effects cannot always be reproduced in clinical series or registry-based studies. ${ }^{12-15} \mathrm{~A}$ key factor in minimizing this risk is to ensure that the study population resembles the true population at risk. In this context, we aim, in this paper, to describe the design and recruitment procedure in the COLOFOL trial, comparing demographic characteristics in randomized patients and in eligible nonparticipants.

\section{Materials and methods}

This study targeted patients who underwent radical surgery for stage II or III sporadic colorectal cancer. To benefit from detection of a recurrence, a patient has to be fit enough to undergo chemotherapy and surgery for metastatic disease. Hence, the trial was restricted to patients $\leq 75$ years of age with a life expectancy exceeding 2 years based on concomitant disease. Complete inclusion and exclusion criteria are provided in Table 1. All patients had to personally sign a written consent before embarking in the study and the trial was approved by the ethical committee of Uppsala University, 2004: M-453 (Sweden) and Copenhagen and Frederikberg Scientific committee, KF 01-194/04 (Denmark).

Requirements for participating as a study center were to recruit at least $30 \%$ of eligible patients and to recruit a total of 25 patients annually. Strict adherence to both requirements
Table I Inclusion and exclusion criteria in the COLOFOL trial

\begin{tabular}{ll}
\hline Inclusion criteria & Exclusion criteria \\
\hline R0 resection & Lynch syndrome or FAP \\
$\begin{array}{l}\text { Age } \leq 75 \text { years } \\
\text { Written informed consent }\end{array}$ & $\begin{array}{l}\text { Local excision (eg, TEM) } \\
\text { Life expectancy }<2 \text { years } \\
\text { Clean colon }\end{array}$ \\
$\begin{array}{l}\text { Inability or refusal to perform informed } \\
\text { consent }\end{array}$ \\
Inability to comply with the control or \\
intense follow-up program
\end{tabular}

Abbreviations: FAP, familial adenomatous polyposis; TEM, transanal endoscopic microsurgery.

proved too difficult for smaller centres and it was later decided to implement a minimum inclusion of 20 patients as cut off to ensure quality. Four centers did not meet these targets and were dropped, leaving 24 recruitment centers in Denmark, Sweden, and Uruguay.

Patients were randomized to either high-frequency or low-frequency follow-up. Both schedules included the same examinations at every follow-up appointment, ie, clinical examination, carcinoembryonic antigen (CEA) test, and computed tomography scan of the liver and thorax. A colonoscopy was required in the perioperative period to verify a clean colon, while further endoscopies were optional. Follow-up occurred at 12 and 36 months postoperatively in the low-frequency arm and at 6,12, 18, 24, and 36 months in the high-frequency arm. Individualized cut-off levels for CEA were used in both arms, based on serum measurements 4 weeks postoperatively. Randomization was computerized in blocks, stratified by center and cancer stage. The size of the blocks was variable and unknown to the participating centers. We considered a $6 \%$ difference in survival as a minimal relevant difference between study arms. We performed a power calculation based on this assumption and estimated 5 -year survival at $60 \%$. With a calculated risk of type 1 error estimated at $5 \%$ and type 2 errors at $15 \%$, we determined that 1,100 patients needed to be randomized in each group. The planned number of randomized patients was set at 2,500, with an expected dropout rate of approximately $20 \%$.

Centers were instructed to register all eligible patients in the web-based study database with a reason for noninclusion, if applicable. In addition, the hospital inpatient rosters of four of the largest participating centers (Aarhus, Bispebjerg, Malmö and Stockholm), accounting for approximately 25\% of all enrolled patients, were examined, in order to identify all patients undergoing colorectal cancer surgery during the recruitment period. Each patient was screened for eligibility, yielding a complete cohort of eligible patients who were not randomized, to serve as controls to the randomized cohort. 
The Mann-Whitney $U$-test was utilized for group comparisons of continuous variables, in which a $P$-value of $<0.05$ was considered statistically significant. Prevalence ratios with $95 \%$ confidence intervals were calculated to compare categorical variables.

\section{Results}

\section{Recruitment}

A total of 13,718 patients underwent surgery for colorectal cancer in the 24 participating centers during the study period. Of these, 5,643 patients met the inclusion criteria. Subsequently, 1,198 (21.0\%) were found to be ineligible, most commonly (34\%) due to a synchronous or previous malignancy. Of the 4,445 remaining eligible patients, 2,509 were randomized (Figure 1). This corresponds to an inclusion rate of $56.4 \%$ among eligible patients, ranging from $17 \%$ to $92 \%$ in the different centers (Figure 2 ). The median number of randomized patients per center was 77 , and the mean number was 107 . The most common reasons for not including eligible patients were as follows: 1) patient was not asked to participate, $n=759(17.1 \%) ; 2)$ patient did not want to participate, $\mathrm{n}=382(8.6 \%) ; 3)$ patient was followed at another hospital, $n=171$ (3.8\%); and 4) other reasons, $n=466(10.5 \%)$. The study flow chart is provided in Figure 1. The recruitment rate was stable, with a median inclusion rate of 43 patients per month (interquartile range: $31-53$ ) (Figure 3).

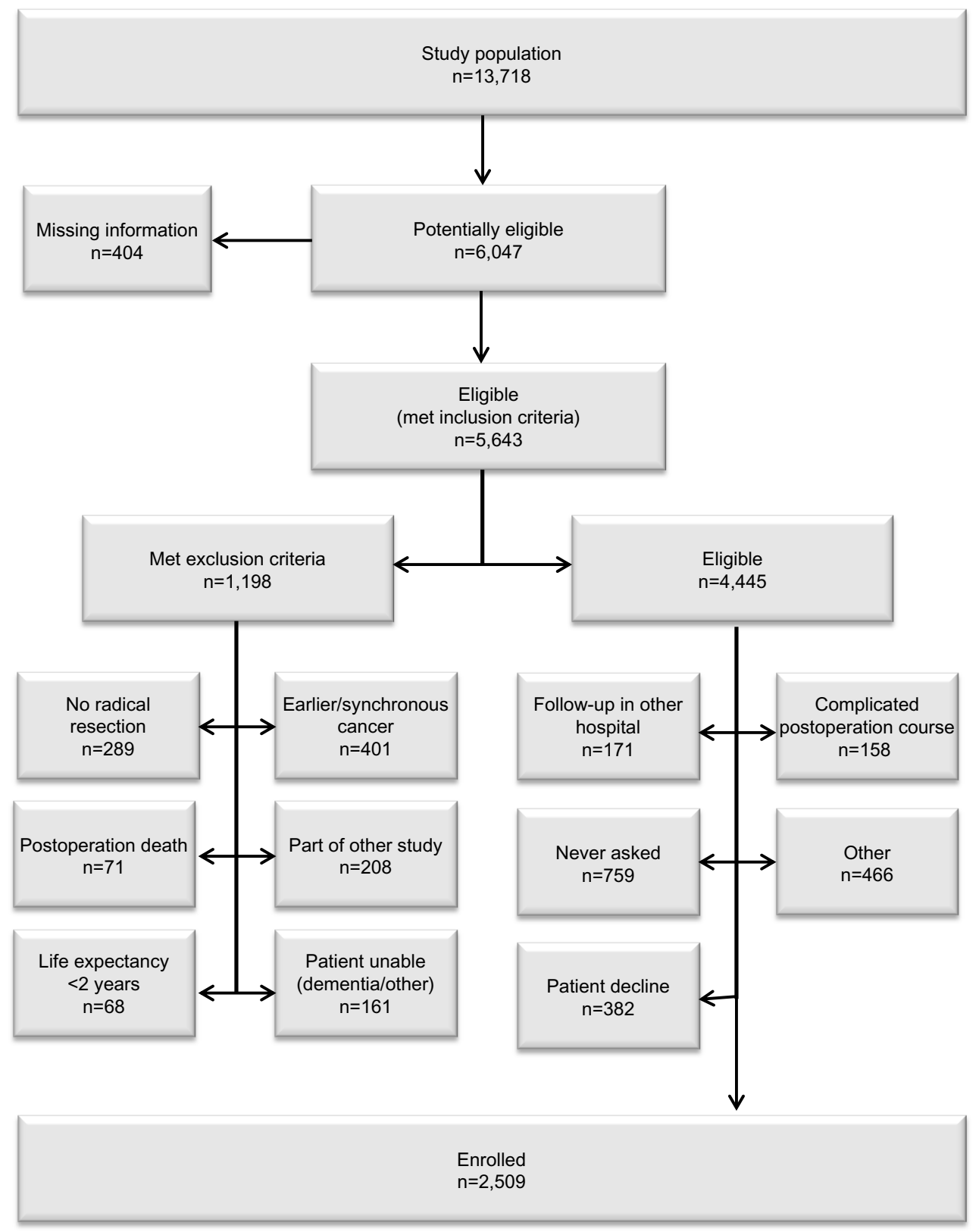

Figure I Consort diagram of the COLOFOL trial. 


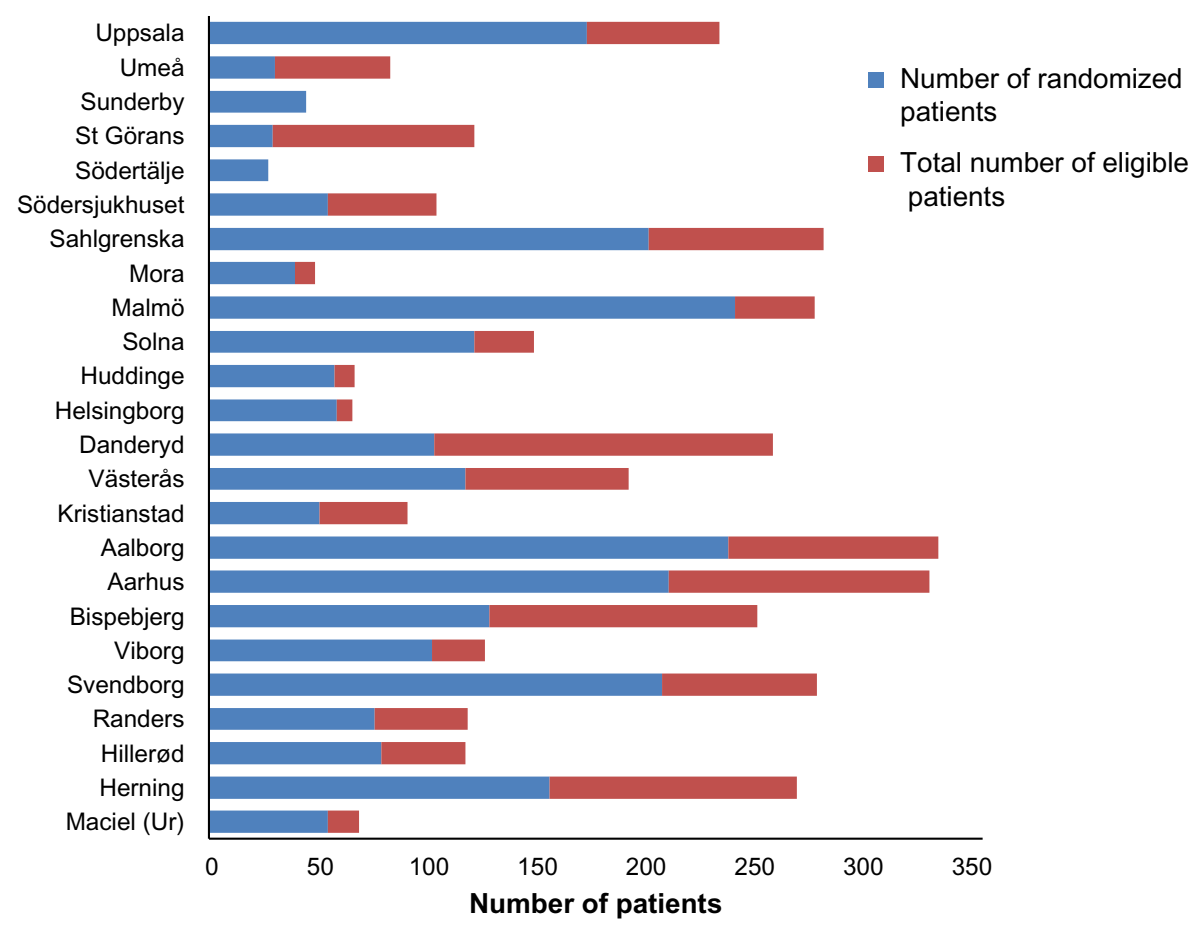

Figure 2 Randomized and eligible patients per center.

At the Aarhus, Bispebjerg, Malmö, and Stockholm centers, a total of 1,221 patients were identified as eligible based on examination of inpatient rosters. Of these, 684 were randomized, corresponding to an inclusion rate of $56 \%$ (range: $46 \%-82 \%$ ). A total of 537 eligible patients were not randomized, among whom 387 (72\%) were reported by study staff and $150(28 \%)$ were missed originally.

\section{Demographics}

In the entire study group $(n=2,509)$, the mean age was 64 years, and the distribution of tumor stage II and III was $54 \%$ and $46 \%$, the male-to-female distribution was $55 \%$ to $45 \%$ and the colon-to-rectum distribution was $65 \%$ to $35 \%$, without any differences between the two study arms.

In the sample of 1,221 patients from the Aarhus, Bispebjerg, Malmö, and Stockholm centers, as described in

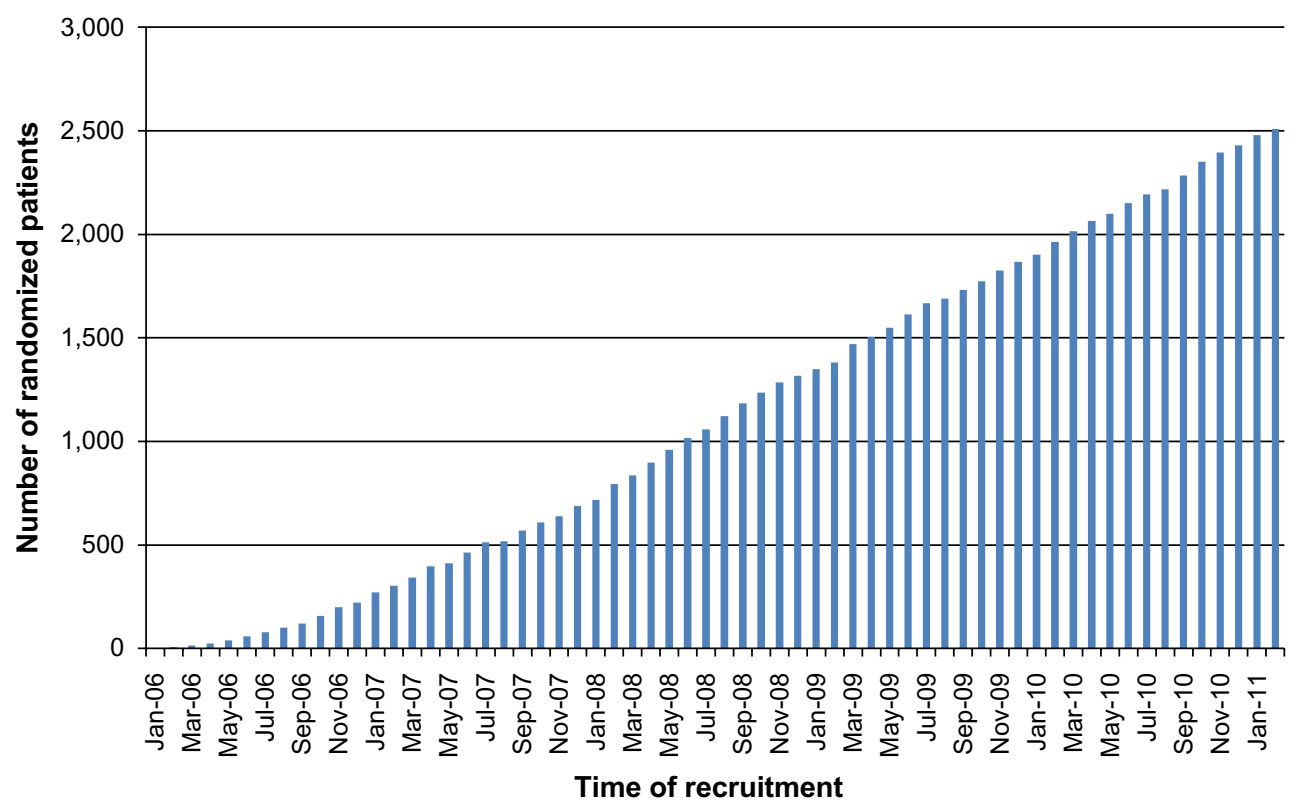

Figure 3 Inclusion rate. 
the Material and methods section, no differences between randomized and nonrandomized patients were observed for age (median age of 65 years in both groups) or sex (60/40\% male-to-female distribution in both groups). These figures were also comparable to the study cohort. However, there were differences in stage distribution and the proportions of colon and rectal cancers, with $6.7 \%$ more stage II disease (prevalence ratio 1.13 [1.02-1.26]) and 5.6\% more colon cancers (prevalence ratio 1.11 [0.99-1.24]) in the randomized group (Table 2).

Patients with stage III disease were younger than patients with stage II disease; however, mean age in stage II and III did not differ significantly between the randomized and nonrandomized groups, 64.5 years and 62.6 years, respectively, in the nonrandomized group compared with 64.1 years and 62.8 years in the randomized group. A higher proportion of elderly ( $>70$ years) was found in the nonrandomized group, both in stage II, 37.0\% compared with $26.4 \%\left(P<0.01, \chi^{2}\right)$ and stage III disease, $28.3 \%$ compared with $20.9 \%\left(P<0.01, \chi^{2}\right)$.

\section{Discussion}

A total of 2,509 patients were randomized in the COLOFOL trial, making it by far the largest randomized trial of follow-up in the field of colorectal cancer. Although this large trial has sufficient power to unveil statistically significant differences, the question arises whether its results can be extrapolated to the target population of colorectal cancer patients. Our comparative analysis of randomized patients and eligible nonparticipants indicated that results of COLOFOL can be generalized.

When designing and performing a study, priorities are to include a well-defined study population based on strict criteria and to rule out the influence of confounders and external contributing factors. However, the risk remains that the results will be difficult to extrapolate to the target population of patients with a given condition. There are several examples of trials whose results could not be reproduced in the general

Table 2 Demography of randomized patients and eligible but nonrandomized patients in four major hospitals, $\mathrm{n}=\mathrm{I}, 22 \mathrm{I}$

\begin{tabular}{lll}
\hline $\begin{array}{l}\text { Demographic } \\
\text { parameters }\end{array}$ & $\begin{array}{l}\text { Randomized } \\
\text { patients (N=684) }\end{array}$ & $\begin{array}{l}\text { Nonrandomized eligible } \\
\text { patients (N=537) }\end{array}$ \\
\hline $\begin{array}{l}\text { Median age, years } \\
\text { (range) }\end{array}$ & $65($ I5-76) & $65($ I5-75) \\
Colon cancer, n (\%) & $385(56)$ & $272(5 \mathrm{I})$ \\
Rectal cancer, n (\%) & $299(44)$ & $264(49)$ \\
Stage II, n (\%) & $383(56)$ & $265(49)$ \\
Stage III, n (\%) & 30 I (44) & $272(5 \mathrm{I})$ \\
Male, n (\%) & $408(60)$ & $323(60)$ \\
Female, n (\%) & $276(40)$ & $214(40)$ \\
\hline
\end{tabular}

population, ${ }^{12,14,15}$ probably due to selection bias regarding comorbidity. Study designs thus represent a delicate balance. ${ }^{11,14}$ In the COLOFOL trial, we chose a pragmatic design with wide inclusion criteria and few exclusion criteria.

A potential trial weakness is that $43.6 \%$ of eligible patients were not randomized. Furthermore, the subanalysis based on hospital inpatient rosters revealed that true number of nonparticipating patients was higher, because only $72 \%$ of nonparticipating patients were originally reported. Our thorough analysis of nonparticipants showed good agreement between eligible nonparticipating patients and randomized patients in regard to age and sex distribution, but moderate, although statistically significant, differences in distribution by cancer stage and localization. The reasons for this are unclear. However, the protocol states that trial analyses will be stratified by stage and localization, which will compensate for these differences (though at the cost of power). No difference in mean age was noted between randomized and nonrandomized patients, whereas a higher proportion of elderly ( $>70$ years) was found in the nonrandomized group. The reason for this is unclear but more comorbidity and inability to consent in this group might contribute. The true percentage of missed eligible patients seems to be higher than the estimated $43.6 \%$. This is of minor importance, because the analysis of eligible nonparticipants indicated that the study cohort was comparable to the source population in respects other than stage and localization. Most likely, differences in localization will not affect the ability to generalize the trial results, because rates of metastasis are similar in colon and rectal cancers. In contrast, stage is the most important risk factor for developing metastases. However, stratification for this parameter should still yield large enough subgroups to permit generalization by stage.

Another shortcoming is a lack of information on comorbidity, except that patients with low life expectancy and patients with American Society of Anesthesiologists' classification of Physical Health score of 4, who were excluded. While randomization ensures that there is no risk for a systematic difference in comorbidity between the study groups, comorbidity can have an unrecognized impact on the generalizability of trial results.

The GILDA trial, another large trial in the field of colorectal cancer, has been recruiting patients since 1998, with the aim of randomizing 1,500 patients. ${ }^{16}$ No results have been published so far indicating a slow inclusion rate. The CEA second look trial in the UK aimed to randomize 2,000 patients, but was stopped after 11 years of recruitment, and 1,474 patients enrolled, due to inability to show any survival benefit from CEA-guided second look 
surgery. ${ }^{17}$ The same negative results for CEA surveillance were reported by Jones et al. ${ }^{18}$ Results from the "Follow-up After Colorectal Surgery" (FACS) trial, in which 1,202 patients were randomized to four different follow-up regimens, were published in 2014. ${ }^{19}$ No advantage was found for any of the regimens. A higher percentage of recurrences were treated with curative intent in the follow-up regimens taken together, compared with minimal follow-up, but no survival benefit was found. Apart from a problem with power, this is probably due to the small possible positive effect, ie, the low rate of asymptomatic recurrences which can be cured by early intervention. One can speculate that this reflects tumor biology, as the correlation between early detection and prognosis is not as strong for metastases as for primary tumors. Compared with the FACS trial, the COLOFOL trial has four times as many patients in each study arm and thus a better chance to detect any difference. Analysis of the final 5-year COLOFOL data will provide strong evidence about the effectiveness of treatment of recurrences with curative intent. Its pragmatic design with wide inclusion criteria and uncomplicated inclusion procedures, reflected by a high and stable monthly recruitment rate, yielded a representative study population and faster recruitment than similar trials. The requirement that at least $30 \%$ of eligible patients be recruited at each center in the COLOFOL trial and its steady recruitment rate have decreased selection biases.

Randomized controlled trials are considered to provide the highest level of scientific evidence. Over 2,500 patients have been randomized in the COLOFOL trial, with a fairly high rate of inclusion. High technical requirements for imaging procedures, verification of a clean colon, standardized algorithms for work-up, and mandatory assessment by multidisciplinary boards enhance the possibility of fair comparison between the study groups. Taken together, all this will permit firm conclusions regarding any benefit on survival of high-frequency compared with low-frequency follow-up after curative surgery for colorectal cancer. Apart from differences in stage and localization (colon or rectum), which will be handled by stratification in the analyses, we found a strong similarity between participants and eligible nonparticipants. This indicates that it will be possible to extrapolate the results to the target population. Three-year data will be available in late 2015 .

\section{Acknowledgments}

The COLOFOL Study Group consists of (in alphabetic order):
Steering committee: Peer Wille-Jørgensen (PI), Bispebjerg University Hospital, Copenhagen, Denmark; Sören Laurberg, Aarhus University Hospital, Denmark; Lars Påhlman, Uppsala Academic Hospital, Sweden; Andrew Renehan, The Christie NHS Foundation Trust, University of Manchester, Manchester Academic Health Science Centre, Manchester UK; Kennet Smedh, Västerås Hospital, Sweden; Ingvar Syk, Skåne University Hospital, Malmö, Sweden; Henrik Toft Sørensen, Aarhus University Hospital, Denmark.

Denmark: Henrik Christensen, Aarhus University Hospital; Per Gandrup, Aalborg University Hospital; Allan Gorm Pedersen, Randers Hospital; Per Jess, Hilleröd Hospital; Mogens Rørbæk Madsen, Herning Hospital; Per Vadgaard Andersen, Svendborg Hospital; Peer Wille-Jørgensen, Bispebjerg University Hospital, Copenhagen; Erling Østergaard, Viborg Hospital.

Sweden: Jonas Bengtsson, Sahlgrenska Hospital, Gothenburg; Mats Bragmark, Danderyd University Hospital; Pamela Buchwald, Helsingborg Hospital; Joakim Folkesson, Uppsala Academic Hospital; Michael Goldinger, St Görans Hospital, Stockholm; Pernilla Hansdotter Andersson, Skåne University Hospital, Malmö; Rolf Heuman, Mora Hospital; Kenneth Lindberg, Södertälje Hospital; Anna Martling, Karolinska Hospital Solna, Stockholm; Michael Dahlberg, Sunderby Hospital, Luleå; Johan Ottosson, Kristianstad Hospital; Birger Sandzén, Norrland University Hospital, Umeå; Monika Egenvall, Karolinska Hospital Huddinge, Stockholm; Kennet Smedh, Västerås Hospital; Parastau Farahnak, Södersjukhuset, Stockholm.

Uruguay: Carlos Barberousse, Maciel University Hospital, Montevideo Uruguay.

This study was supported by unregistered grants from the Nordic Cancer Union, A.P. Møller Foundation, Beckettfoundation, Grosserer Chr. Andersen og hustru bursary, Sigvald og Edith Rasmussens Memorial Fund, Martha Margrethe og Christian Hermansens Fund, the Danish Medical Association, the Danish Cancer Society, the Danish Council for Independent Research/Medical Sciences and Swedish Cancer Foundation project CAN 2013/553.

\section{Disclosure}

The authors report no conflicts of interest in this work.

\section{References}

1. Baca B, Beart RW Jr, Etzioni DA. Surveillance after colorectal cancer resection: a systematic review. Dis Colon Rectum. 2011;54(8): 1036-1048.

2. Komborozos VA, Skrekas GJ, Pissiotis CA. The contribution of follow-up programs in the reduction of mortality of rectal cancer recurrences. Dig Surg. 2001;18(5):403-408. 
3. Rodriguez-Moranta F, Salo J, Arcusa A, et al. Postoperative surveillance in patients with colorectal cancer who have undergone curative resection: a prospective, multicenter, randomized, controlled trial. $J$ Clin Oncol. 2006;24(3):386-393.

4. Secco GB, Fardelli R, Gianquinto D, et al. Efficacy and cost of risk-adapted follow-up in patients after colorectal cancer surgery: a prospective, randomized and controlled trial. Eur J Surg Oncol. 2002;28(4):418-423.

5. Makela JT, Laitinen SO, Kairaluoma MI. Five-year follow-up after radical surgery for colorectal cancer. Results of a prospective randomized trial. Arch Surg. 1995;130(10):1062-1067.

6. Ohlsson B, Breland U, Ekberg H, Graffner H, Tranberg KG. Follow-up after curative surgery for colorectal carcinoma. Randomized comparison with no follow-up. Dis Colon Rectum. 1995;38(6):619-626.

7. Kjeldsen BJ, Kronborg O, Fenger C, Jorgensen OD. A prospective randomized study of follow-up after radical surgery for colorectal cancer. Br J Surg. 1997;84(5):666-669.

8. Pietra N, Sarli L, Costi R, Ouchemi C, Grattarola M, Peracchia A. Role of follow-up in management of local recurrences of colorectal cancer: a prospective, randomized study. Dis Colon Rectum. 1998; 41(9):1127-1133.

9. Jeffery GM, Hickey BE, Hider P. Follow-up strategies for patients treated for non-metastatic colorectal cancer. Cochrane Database Syst Rev. 2002;1:CD002200.

10. Renehan AG, Egger M, Saunders MP, O’Dwyer ST. Impact on survival of intensive follow up after curative resection for colorectal cancer: systematic review and meta-analysis of randomised trials. BMJ. 2002; 324(7341):813.

11. Hulley SB, Cummings SR, Browner WS. Designing Clinical Research: An Epidemiologic Approach. Baltimore: Williams \& Wilkins; 1988:xi, 247.
12. Sorbye H, Pfeiffer P, Cavalli-Bjorkman N, et al. Clinical trial enrollment, patient characteristics, and survival differences in prospectively registered metastatic colorectal cancer patients. Cancer. 2009; 115(20):4679-4687.

13. Peppercorn JM, Weeks JC, Cook EF, Joffe S. Comparison of outcomes in cancer patients treated within and outside clinical trials: conceptual framework and structured review. Lancet. 2004;363(9405):263-270.

14. Sorensen HT, Lash TL, Rothman KJ. Beyond randomized controlled trials: a critical comparison of trials with nonrandomized studies. Hepatology. 2006;44(5):1075-1082.

15. Hansen AB, Gerstoft J, Kirk O, et al. Unmeasured confounding caused slightly better response to HAART within than outside a randomized controlled trial. J Clin Epidemiol. 2008;61(1):87-94.

16. Grossmann EM, Johnson FE, Virgo KS, Longo WE, Fossati R. Follow-up of colorectal cancer patients after resection with curative intent-the GILDA trial. Surg Oncol. 2004;13(2-3):119-1124.

17. Treasure T, Monson K, Fiorentino F, Russell C. The CEA secondlook trial: a randomised controlled trial of carcinoembryonic antigen prompted reoperation for recurrent colorectal cancer. BMJOpen. 2014 4(5):e004385.

18. Jones RP, McWhirter D, Fretwell VL, McAvoy A, Hardman JG. Clinical follow-up does not improve survival after resection of stage I-III colorectal cancer: a cohort study. Int J Surg. 2015;17:67-71.

19. Primrose JN, Perera R, Gray A, Rose P, Fuller A, Corkhill A, et al Effect of 3 to 5 years of scheduled CEA and CT follow-up to detect recurrence of colorectal cancer: the FACS randomized clinical trial. JAMA. 2014;311(3):263-270.
Clinical Epidemiology

\section{Publish your work in this journal}

Clinical Epidemiology is an international, peer-reviewed, open access, online journal focusing on disease and drug epidemiology, identification of risk factors and screening procedures to develop optimal preventative initiatives and programs. Specific topics include: diagnosis, prognosis, treatment, screening, prevention, risk factor modification,

Submit your manuscript here: http://www.dovepress.com/clinical-epidemiology-journa

\section{Dovepress}

systematic reviews, risk \& safety of medical interventions, epidemiology \& biostatistical methods, and evaluation of guidelines, translational medicine, health policies \& economic evaluations. The manuscript management system is completely online and includes a very quick and fair peer-review system, which is all easy to use. 